\title{
ON THE SPECTRAL PROPERTIES AND STABILIZATION OF ACOUSTIC FLOW
}

\author{
WALTER LitTMan Bo LiU
}

School of Math., University of Minnesota, Minneapolis, MN55455

\begin{abstract}
In this paper we use perturbation theory to study the spectral properties and energy decay of two dimensional acoustic flow (cf. Beale[1], Morse \& Ingard[10]): $\phi_{t t}-c^{2} \Delta \phi=0$ in $\Omega \times(0, \infty), m \delta_{t t}+d \delta_{t}+k \delta=-\rho \phi_{t}$ and $\phi_{x}=\delta_{t}$ on $\Gamma_{0} \times(0, \infty), \frac{\partial \phi}{\partial \nu}=0$ on $\Gamma_{1} \times(0, \infty)$ with initial data $\phi(0)=\phi_{0}, \phi_{t}(0)=\phi_{1}$ in $\Omega$ and $\delta(0)=\delta_{0}, \delta_{t}(0)=\delta_{1}$ on $\Gamma_{0}$ where $\Omega=(0,1) \times(0,1), \Gamma_{0}=\{(1, y) ; 0<$ $y<1\}, \Gamma_{1}=\partial \Omega \backslash \Gamma_{0}$ and $\nu$ is the external normal direction on the boundary. Locations of eigenvalues of the infinitesimal generator of semigroup associated with the above system are estimated. A certain "Fourier" expansion are obtained. That the energy decays to zero and like $t^{-1}$ (even like $t^{-\beta}$ ) if initial data satisfy certain smoothness are proved.
\end{abstract}

\section{Introduction.}

In this paper we study the energy decay and the related spectral properties of the following two dimensional acoustic flow model: Let $\Omega=(0,1) \times(0,1)$ be filled with fluid which is at rest except for acoustic wave motion. Let $\phi$ be the velocity potential so that $-\nabla \phi$ is the particle velocity. Assume that the sides $y=0,1$ and $x=0$ are rigid and impenetrable to that kind of fluid so that $\frac{\partial \phi}{\partial \nu}=0$ where $\nu$ is a unit exterior normal vector. Assume that the side $x=1$ is not rigid but subject to small oscillations (like a container of the rectangular shape sealed on one side by a resilient material). As in Beale[1]'s model we assume that each point on the side $x=1$ reacts to the excess pressure $\rho \phi_{t}$ (where $\rho$ is the density of the fluid) of the acoustic wave like a resistive harmonic oscillator; and that we also assume that the different parts of the side $x=1$ do not influence each

1991 Mathematics Subject Classification. 35B40, 35C20, 35L20, 35P20, 49 N35.

Typeset by $\mathcal{A} \mathcal{M S}-\mathrm{T}_{\mathrm{E}} \mathrm{X}$ 
other and hence the tangential oscillations (deformations) can be ignored. Let $\delta(y, t)$ be the normal displacement of the side $x=1$. Let $\mathrm{c}$ denote the speed of sound in the medium. We also assume that the side $x=1$ is impenetrable, i.e. $\phi_{x}=\delta_{t}$ on $x=1$. Then the motion of such system is governed by the following partial differential equations:

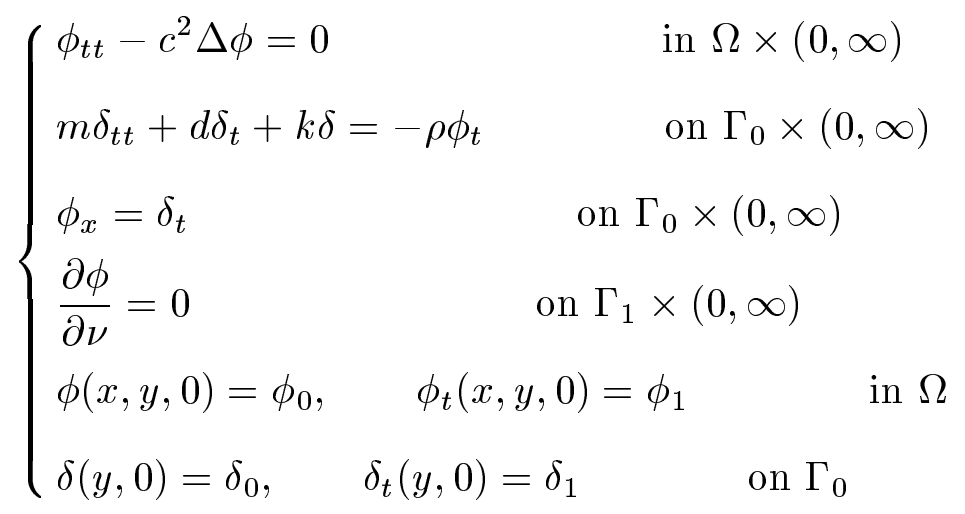

where $\Gamma_{0}=(1, y), \Gamma_{1}=\partial \Omega \backslash \Gamma_{0}$ and $m, d, k$ are physical constants and positive. This model is similar to three dimensional model used as an example in Morse \& Ingard[10] for waves assumed to be at a definite frequency (cf. pg.263 and also Beale[1]). The energy associated with the system (1.1) is defined as

$$
\mathcal{E}(t)=\frac{1}{2} \iint_{\Omega}\left[\rho|\nabla \phi|^{2}+\rho c^{-2} \phi_{t}^{2}\right] d x d y+\frac{1}{2} \int_{\Gamma_{0}}\left[k \delta^{2}+m \delta_{t}^{2}\right] d y .
$$

It follows from integration by parts that one has

$$
\frac{d \mathcal{E}(t)}{d t}=-\int_{\Gamma_{0}} d \delta_{t}^{2} d y
$$

for appropriate smooth solutions, $\phi$ and $\delta$, of the system (1.1). Thus we see that the energy of the system (1.1) decays if $d>0$. In this paper we shall study how the energy of the system (1.1) decays. Micu \& Zuazua[8][9] respectively have shown that the energy of the system (1.1) decays to zero when $d \delta_{t}+k \delta$ in the second equation of the system (1.1) is replaced by $\delta_{t}-\delta_{y y}$ or $-\delta_{y y t}-\delta_{y y}$. However it is very difficult even if one trys to show that the energy of the system 
(1.1) decays to zero by $\omega$-limit theory (cf. Hale[3]) because the infinitesimal generator of the semigroup associated with the system (1.1) in a Hilbert space no longer has compact resolvent. We here study the spectral properties of the infinitesimal generator of the semigroup associated with the system (1.1) by perturbation theory and then we use these results to attack the energy decay of the system (1.1) and to obtain rational decay rates (i.e. like $t^{-\beta}$ ) of the energy of (1.1) (rather than that the energy just decays to zero) when the initial data has certain smoothness. Such idea was applied successfully by Littman \& Markus[6][7] to the energy decay rate of the equations for either Euler-Bernoulli beam or string attached to a movable mass at one end of the beam or string.

Usually the system (1.1) can be solved by semigroup theory in a Hilbert space. Let $H_{c}(\Omega)$ denote the quotient space of complex $H^{1}(\Omega)$ modulo constants. Let $\mathcal{H}=H_{c}(\Omega) \times L^{2}(\Omega) \times L^{2}\left(\Gamma_{0}\right) \times L^{2}\left(\Gamma_{0}\right)$ be the complex Hilbert space of elements $\left(\phi, \phi_{t}, \delta, \delta_{t}\right)$ with the associated inner product

$$
\langle\vec{f}, \vec{g}\rangle_{\mathcal{H}}=\iint_{\Omega} \rho\left[\nabla f^{(1)} \cdot \overline{\nabla g^{(1)}}+c^{-2} f^{(2)} \overline{g^{(2)}}\right] d x d y+\int_{\Gamma_{0}}\left[k f^{(3)} \overline{g^{(3)}}+m f^{(4)} \overline{g^{(4)}}\right] d y
$$

where $\vec{f}=\left(f^{(1)}, f^{(2)}, f^{(3)}, f^{(4)}\right), \quad \vec{g}=\left(g^{(1)}, g^{(2)}, g^{(3)}, g^{(4)}\right) \in \mathcal{H}$. It is easy to see, by the Poincaré inequality, that (1.4) is well defined. The associated norm on the Hilbert space $\mathcal{H}$ is denoted by $\|\cdot\|_{\mathcal{H}}$. For the sake of convenience we write column vector as row vector throughout this paper. We now define an operator $\mathcal{A}: \mathcal{D}(\mathcal{A}) \subset \mathcal{H} \rightarrow \mathcal{H}$ by

$$
\mathcal{A} \vec{f}=\left(f^{(2)}, c^{2} \Delta f^{(1)}, f^{(4)}, \frac{-1}{m}\left(\rho f^{(2)}+k f^{(3)}+d f^{(4)}\right)\right)
$$

where $\mathcal{D}(\mathcal{A})=\left\{\left(f^{(1)}, f^{(2)}, f^{(3)}, f^{(4)}\right) \in \mathcal{H} ; \Delta f^{(1)} \in L^{2}(\Omega), \quad f^{(2)} \in H^{1}(\Omega), \frac{\partial f^{(1)}}{\partial \nu}\right.$ $=0$ on $\Gamma_{1}$ and $\frac{\partial f^{(1)}}{\partial x}=f^{(4)}$ on $\left.\Gamma_{0}\right\}$. Here $f^{(2)}$ in the last component of $\mathcal{A} \vec{f}$ should be understood as the trace of $f^{(2)}$ on $\Gamma_{0}$. The conditions $\frac{\partial f^{(1)}}{\partial \nu}=$ 0 on $\Gamma_{1}$ and $\frac{\partial f^{(1)}}{\partial x}=f^{(4)}$ on $\Gamma_{0}$ are interpreted in the following weak sense

$$
\iint_{\Omega}\left[\Delta f^{(1)} \bar{v}+\nabla f^{(1)} \cdot \nabla \bar{v}\right] d x d y=\int_{\Gamma_{0}} f^{(4)} \bar{v} d y \quad \forall v \in H^{1}(\Omega) .
$$


It is well known that $f^{(1)} \in H^{2}(\Omega)$ if and only if $f^{(4)} \in H^{1 / 2}\left(\Gamma_{0}\right)$ and $\Delta f^{(1)} \in$ $L^{2}(\Omega)$. Let $\vec{u}=\left(\phi, \phi_{t}, \delta, \delta_{t}\right)$. Then the system (1.1) is equivalent to the following evolution equation in the Hilbert space $\mathcal{H}$.

$$
\begin{cases}\vec{u}_{t}=\mathcal{A} \vec{u}, & \vec{u} \in \mathcal{H} \\ \vec{u}(0)=\left(\phi_{0}, \phi_{1}, \delta_{0}, \delta_{1}\right) . & \end{cases}
$$

Using integration by parts, we know that the operator $\mathcal{A}$ has the dissipative property, that is

$$
\operatorname{Re}\langle\mathcal{A} \vec{f}, \vec{f}\rangle_{\mathcal{H}}=-\int_{\Gamma_{0}} d\left|f^{(4)}\right|^{2} d x
$$

Using this property (1.7) and an argument similar to the one used in the proof of theorem 2.1 of Beale[1], one can easily show

Proposition 1. $\mathcal{A}$ is a closed, densely defined and dissipative operator. It is an infinitesimal generator of a $C_{0}$-contraction semigroup. If $d=0$, then $\mathcal{A}$ is skew-adjoint and an infinitesimal generator of a unitary semigroup.

Remark 1.1: $\mathcal{A}$ does not have compact resolvent due to the fact that the mapping from $\vec{f}$ to the third component of $\mathcal{A}^{-1} \vec{f}$ is not compact.

Proposition 1 tells us that the system (1.1) allows a unique solution $(\phi, \delta) \in$ $C\left(0, \infty ; H^{1}(\Omega) \times L^{2}\left(\Gamma_{0}\right)\right) \times C^{1}\left(0, \infty ; L^{2}(\Omega) \times L^{2}\left(\Gamma_{0}\right)\right)$ if $\vec{u}(0) \in \mathcal{H}$.

The outline of this paper is as follows: In $\S 2$ we estimate the location of all of eigenvalues of the operator $\mathcal{A}$. In $\S 3$ we shall prove that for sufficiently small $d$, any function in $\mathcal{H}$ has a certain "Fourier" expansion based on a collection of eigenfunctions of $\mathcal{A}$ and a projection on a subspace which is the closure of the span of another collection of generalized eigenfunctions of $\mathcal{A}$. This result improves a two-dimensional version of Beale[1]'s three dimensional result, according to which the span of all generalized eigenfunctions of the infinitesimal 
generator $\mathcal{A}$ is dense in the corresponding Hilbert space. Finally in $\S 4$ we shall show that the energy of the system (1.1) decays at rational rates if the initial data of the system (1.1) satisfy certain smoothness conditions.

\section{Estimates of Location of Eigenvalues.}

It is easy to see that $\mathcal{A}^{-1}$ is a bounded operator from $\mathcal{H}$ to $\mathcal{H}$. With (1.7), we know that all of eigenvalues of the operator $\mathcal{A}$ stay in the strip $-\kappa_{0} \leq x \leq 0$ in the complex plane for some $\kappa_{0}>0$. How are those eigenvalues located in this strip? Beale[1](theorem 4.2) has shown that $\operatorname{Re}(\lambda) \rightarrow 0$ when $|\operatorname{Im}(\lambda)| \rightarrow \infty$ for the eigenvalue $\lambda$ of $\mathcal{A}$ if $\Omega$ is a bounded open domain in $R^{3}$ and $\Gamma_{0}=\partial \Omega$. By the same argument as in the proof of theorem 4.2 of Beale[1] one can show that this result for the operator $\mathcal{A}$ of the system (1.1) still holds but we can estimate how fast the eigenvalue $\lambda$ of $\mathcal{A}$ approaches the imaginary axis in the complex plane when $|\lambda|$ goes to $+\infty$. Let $\lambda \neq 0$ be an eigenvalue of the operator $\mathcal{A}$ and $\vec{\psi}=\left(\psi^{(1)}, \psi^{(2)}, \psi^{(3)}, \psi^{(4)}\right) \in \mathcal{H}$ be an eigenfunction of $\mathcal{A}$ corresponding to the eigenvalue $\lambda$. Then we have that $\vec{\psi}=\left(\psi^{(1)}, \psi^{(2)}, \psi^{(3)}, \psi^{(4)}\right) \in \mathcal{H}$ satisfy

$$
\left\{\begin{array}{l}
-c^{2} \Delta \psi^{(1)}+\lambda^{2} \psi^{(1)}=0 \quad \text { in } \Omega \\
\left(m \lambda^{2}+d \lambda+k\right) \frac{\partial \psi^{(1)}}{\partial x}+\rho \lambda^{2} \psi^{(1)}=0 \quad \text { on } \Gamma_{0} \\
\frac{\partial \psi^{(1)}}{\partial \nu}=0 \quad \text { on } \Gamma_{1}
\end{array}\right.
$$

and

$$
\left\{\begin{array}{l}
\psi^{(2)}=\lambda \psi^{(1)} \quad \text { in } \Omega \\
\psi^{(4)}=\frac{\partial \psi^{(1)}}{\partial x}, \quad \psi^{(3)}=\frac{1}{\lambda} \psi^{(4)} \quad \text { on } \Gamma_{0} .
\end{array}\right.
$$

It is easy to check that $\vec{\psi}$ is smooth. Since the operator $\frac{\partial^{2}}{\partial y^{2}}$ with homogeneous Neumann boundary conditions at $y=0,1$ is self-adjoint and has compact resolvent, eigenvalue problem (2.1) is solvable by the method of separating vari- 
ables. Let $\psi^{(1)}=\chi(x) \cos (n \pi y)$. Then $\chi(x)$ satisfies

$$
\left\{\begin{array}{l}
-c^{2} \chi^{\prime \prime}(x)+\left[\lambda^{2}+c^{2}(n \pi)^{2}\right] \chi(x)=0 \quad x \in(0,1) \\
\chi^{\prime}(0)=0, \quad\left(m \lambda^{2}+d \lambda+k\right) \chi^{\prime}(1)+\rho \lambda^{2} \chi(1)=0
\end{array}\right.
$$

The general solution of the first two equations of eigenvalue problem (2.3) is

$$
\chi(x)=C \cosh (\xi x), \quad x \in(0,1) .
$$

Imposing the last equation of (2.3), we find that $\xi$ and $\lambda$ must satisfy

$$
\left\{\begin{array}{l}
\xi\left(m \lambda^{2}+d \lambda+k\right) \sinh \xi+\rho \lambda^{2} \cosh \xi=0 \\
\xi=\frac{1}{c} \sqrt{\lambda^{2}+c^{2}(n \pi)^{2}}
\end{array}\right.
$$

We use perturbation theory to study the solutions of equations (2.5). We first investigate the solutions of equations (2.5) for the case $d=0$ in which case (by proposition 1) we know that the solutions $\lambda$ of equations (2.5) correspond with the eigenvalues of a skew-adjoint operator on the Hilbert space $\mathcal{H}$. Then all $\lambda$ for the case $d=0$ have only imaginary part and are located symmetrically on the imaginary axis. Thus $\xi$ is either a pure imaginary number (if $\lambda^{2}+c^{2}(n \pi)^{2}<$ 0 ) or a positive real number (if $\lambda^{2}+c^{2}(n \pi)^{2}>0$ ). But from the first equation of (2.5) we know that $\xi$ can not be a positive real number if $d=0$ and $m \lambda^{2}+k \leq 0$. Let $\hat{\xi}_{j, n}$ denote the $j$-th positive solution of equation

$$
\xi\left\{-m c^{2}\left[\xi^{2}+(n \pi)^{2}\right]+k\right\} \sin \xi+\rho c^{2}\left[\xi^{2}+(n \pi)^{2}\right] \cos \xi=0 .
$$

Lemma 1. If $d=0$, then there are positive constants $d_{1}$ and $d_{2}$ independent of parameters $j$ and $n$ such that

$$
j \pi+\frac{d_{1}}{j} \leq \hat{\xi}_{j+1, n} \leq j \pi+\frac{d_{2}}{j} \quad \text { for } j^{2}+n^{2} \text { sufficiently large and } j \geq 1 \text {. }
$$


Proof. For any positive constant $d^{\star}$, consider the auxiliary equation

$$
d^{\star} \xi=\cot \xi
$$

Let $\xi_{j}^{\star}$ be the $j$-th positive solution of equation (2.8). Using the substitution $\xi^{\prime}=\xi-j \pi$ and the inequality $\frac{1}{2 \xi^{\prime}} \leq \cot \xi^{\prime} \leq \frac{1}{\xi^{\prime}}$ for $\xi^{\prime} \in\left(0, \frac{\pi}{4}\right]$, we have $\frac{1}{2} \leq d^{\star} \xi_{j+1}^{\star}\left(\xi_{j+1}^{\star}-j \pi\right) \leq 1$ for $j$ sufficiently large (depending on $\left.d^{\star}\right)$. Using the fact that $1+\frac{1}{3} \xi^{\prime} \leq \sqrt{1+\xi^{\prime}} \leq 1+\frac{1}{2} \xi^{\prime}$ for $\xi^{\prime} \in[0,1]$ and the above quadratic inequality, we have that $\xi_{j+1}^{\star}$ satisfies

$$
j \pi+\frac{d_{1}\left(d^{\star}\right)}{j \pi} \leq \xi_{j+1}^{\star} \leq j \pi+\frac{d_{2}\left(d^{\star}\right)}{j \pi} \quad \text { for } j \geq 1
$$

where $d_{1}\left(d^{\star}\right)$ and $d_{2}\left(d^{\star}\right)$ are constants independent of $j$.

Since $0<d_{1}^{\star} \leq \frac{m c^{2}\left[\xi^{2}+(n \pi)^{2}\right]-k}{\rho c^{2}\left[\xi^{2}+(n \pi)^{2}\right]}<d_{2}^{\star}$ for some constants $d_{1}^{\star}$ and $d_{2}^{\star}$ independent of parameters $\xi$ and $n$ when $\xi^{2}+(n \pi)^{2}$ is large, we have that $\hat{\xi}_{j, n}$ lies between the $j^{t h}$ positive solution $\xi_{j}^{(2)}$ of equation (2.8) for $d^{\star}=d_{2}^{\star}$ and the $j^{\text {th }}$ positive solution $\xi_{j}^{(1)}$ of equation (2.8) for $d^{\star}=d_{1}^{\star}$. Thus lemma 1 holds and (2.7) follows from (2.9). $\quad$ Q.E.D.

Remark 2.1: $\hat{\xi}_{1, n}$ for sufficiently large $n$ are also uniformly bounded below away from zero.

Let $\hat{\lambda}_{j, n}=c \sqrt{\hat{\xi}_{j, n}^{2}+(n \pi)^{2}} i$ where the $\hat{\xi}_{j, n}$ are as above. From the above argument we know that the eigenvalue $\lambda$ of the operator $\mathcal{A}$ for the case $d=0$ has only forms $\pm \hat{\lambda}_{j, n}$ when $|\lambda|$ is sufficiently large. From now throughout this paper $C$ always denotes a constant independent of parameters unless we mention specifically what important parameters it depends on

Theorem 1. There are positive constants $\lambda^{\star}$ and $\alpha^{\star}\left(\lambda^{\star}\right)$ such that if $d \leq \alpha^{\star}$ and $\left|\hat{\lambda}_{j, n}\right| \geq \lambda^{\star}$, then there is a solution $\lambda_{j, n}$ of (2.5) such that

$$
\left|\lambda_{j, n}-\hat{\lambda}_{j, n}\right| \leq C\left[\hat{\xi}_{j, n}^{2}+(n \pi)^{2}\right]^{-1}
$$


Moreover let $\xi_{j, n}=\frac{1}{c} \sqrt{\lambda_{j, n}^{2}+c(n \pi)^{2}}$, then

$$
\left|\xi_{j, n}-\hat{\xi}_{j, n} i\right| \leq C\left[\hat{\xi}_{j, n}^{2}+(n \pi)^{2}\right]^{-\frac{1}{2}} \hat{\xi}_{j, n}^{-1}
$$

Proof. Consider the mapping $\xi(\lambda)=\frac{1}{c} \sqrt{\lambda^{2}+c^{2}(n \pi)^{2}}$. We know that the above mapping is an isomorphism from $\mathcal{U}_{\lambda}$, the sector $\theta \in\left(0, \frac{3 \pi}{4}\right)$ with the vertex at $(0,0)$ in the complex $\lambda$-plane cut along the imaginary axis from 0 to $c n \pi i$, onto $\mathcal{U}_{\xi}$, an open set of the upper complex $\xi$-plane above the curve $(\operatorname{Re}(\xi))^{2}-$ $(\operatorname{Im}(\xi))^{2}=1$ for $\operatorname{Re}(\xi) \leq 0$. Moreover we have $\xi\left(\hat{\lambda}_{j, n}\right)=\hat{\xi}_{j, n} i$. Let $\gamma_{j}^{\xi}$ be a circle in the complex $\xi$-plane with the center at $\hat{\xi}_{j, n} i$ and radius $r$ which is very small and will be determined later in the proof. We assume that $j^{2}+n^{2}$ is very large so that $\gamma_{j}^{\xi}$ lies in $\mathcal{U}_{\xi}$. We denote by $\gamma_{j, n}^{\lambda}$ the closed curve $\xi^{-1}\left(\gamma_{j}^{\xi}\right)$ in $\mathcal{U}_{\lambda}$. Let $\xi=\hat{\xi}_{j, n} i+r e^{i \theta}$ be a point on $\gamma_{j}^{\xi}$ and $\lambda \in \gamma_{j, n}^{\lambda}$ such that $\xi(\lambda)=\xi$. Let

$$
\begin{aligned}
& f(\xi)=\left\{m c^{2}\left[\xi^{2}-(n \pi)^{2}\right]+k\right\} \sinh \xi+\rho c^{2}\left[\xi-\frac{(n \pi)^{2}}{\xi}\right] \cosh \xi \\
& g(\xi)=d c \sqrt{\xi^{2}-(n \pi)^{2}} \sinh \xi
\end{aligned}
$$

It is well-known that the following identities hold for any complex numbers $z_{1}$ and $z_{2}$.

$$
\begin{aligned}
& \sinh \left(z_{1}+z_{2}\right)=\sinh \left(z_{1}\right) \cosh \left(z_{2}\right)+\cosh \left(z_{1}\right) \sinh \left(z_{2}\right) \\
& \cosh \left(z_{1}+z_{2}\right)=\cosh \left(z_{1}\right) \cosh \left(z_{2}\right)+\sinh \left(z_{1}\right) \sinh \left(z_{2}\right) .
\end{aligned}
$$

Substituting $\xi=\hat{\xi}_{j, n} i+r e^{i \theta} \in \gamma_{j}^{\xi}$ into (2.12) and using (2.13), we have (after 
careful calculation)

$$
\begin{aligned}
& \operatorname{Re}(f(\xi))=\left\{m c^{2}\left[r^{2} \cos ^{2} \theta-\left(r \sin \theta+\hat{\xi}_{j, n}\right)^{2}-(n \pi)^{2}\right]+k\right\} . \\
& \text { - }\left[\cos \hat{\xi}_{j, n} \sinh (r \cos \theta) \cos (r \sin \theta)-\sin \hat{\xi}_{j, n} \sinh (r \cos \theta) \sin (r \sin \theta)\right] \\
& -2 m c^{2} r \cos \theta\left(r \sin \theta+\hat{\xi}_{j, n}\right)\left[\cos \hat{\xi}_{j, n} \cosh (r \cos \theta) \sin (r \sin \theta)\right. \\
& \left.+\sin \hat{\xi}_{j, n} \cosh (r \cos \theta) \cos (r \sin \theta)\right]+\rho c^{2}\left[1-\frac{(n \pi)^{2}}{|\xi|^{2}}\right] r \cos \theta \\
& \text { - }\left[\cos \hat{\xi}_{j, n} \cosh (r \cos \theta) \cos (r \sin \theta)-\sin \hat{\xi}_{j, n} \cosh (r \cos \theta) \sin (r \sin \theta)\right] \\
& -\rho c^{2}\left[1+\frac{(n \pi)^{2}}{|\xi|^{2}}\right]\left(r \sin \theta+\hat{\xi}_{j, n}\right) \cdot \\
& \text { - }\left[\cos \hat{\xi}_{j, n} \sinh (r \cos \theta) \sin (r \sin \theta)+\sin \hat{\xi}_{j, n} \sinh (r \cos \theta) \cos (r \sin \theta)\right] \\
& =I_{r e}^{1}+I_{r e}^{2}+I_{r e}^{3}+I_{r e}^{4}, \\
& \operatorname{Im}(f(\xi))=\left\{m c^{2}\left[r^{2} \cos ^{2} \theta-\left(r \sin \theta+\hat{\xi}_{j, n}\right)^{2}-(n \pi)^{2}\right]+k\right\} \\
& \text { - }\left[\cos \hat{\xi}_{j, n} \cosh (r \cos \theta) \sin (r \sin \theta)+\sin \hat{\xi}_{j, n} \cosh (r \cos \theta) \cos (r \sin \theta)\right] \\
& +2 m c^{2} r \cos \theta\left(r \sin \theta+\hat{\xi}_{j, n}\right)\left[\cos \hat{\xi}_{j, n} \sinh (r \cos \theta) \cos (r \sin \theta)\right. \\
& \left.-\sin \hat{\xi}_{j, n} \sinh (r \cos \theta) \sin (r \sin \theta)\right]+\rho c^{2}\left[1-\frac{(n \pi)^{2}}{|\xi|^{2}}\right] r \cos \theta \\
& \cdot\left[\cos \hat{\xi}_{j, n} \sinh (r \cos \theta) \sin (r \sin \theta)+\sin \hat{\xi}_{j, n} \sinh (r \cos \theta) \cos (r \sin \theta)\right] \\
& +\rho c^{2}\left[1+\frac{(n \pi)^{2}}{|\xi|^{2}}\right]\left(r \sin \theta+\hat{\xi}_{j, n}\right) \text {. } \\
& \text { - }\left[\cos \hat{\xi}_{j, n} \cosh (r \cos \theta) \cos (r \sin \theta)-\sin \hat{\xi}_{j, n} \cosh (r \cos \theta) \sin (r \sin \theta)\right] \\
& =I_{i m}^{1}+I_{i m}^{2}+I_{i m}^{3}+I_{i m}^{4} \text {. }
\end{aligned}
$$

From (2.5) we have

$$
\left|\lambda-\hat{\lambda}_{j, n}\right| \leq \frac{C \hat{\xi}_{j, n}}{\sqrt{\hat{\xi}_{j, n}^{2}+(n \pi)^{2}}} r \quad \forall \lambda \in \gamma_{j, n}^{\lambda} .
$$

Using (2.7), (2.12), (2.13) and (2.16), we have

$$
|g(\xi)| \leq C d\left[\hat{\xi}_{j, n}^{2}+(n \pi)^{2}\right]^{\frac{1}{2}}\left[r+\frac{1}{\hat{\xi}_{j, n}}\right] \quad \forall \xi \in \gamma_{j}^{\xi} .
$$


Now we choose

$$
r=\left[\hat{\xi}_{j, n}^{2}+(n \pi)^{2}\right]^{-\frac{1}{2}} \hat{\xi}_{j, n}^{-1}
$$

Using (2.7), $\hat{\xi}_{j, n} \sin \hat{\xi}_{j, n} \sim \cos \hat{\xi}_{j, n}$ and the Taylor expansion, we have

$$
\begin{aligned}
& I_{r e}^{1}=-m c^{2} \cos \hat{\xi}_{j, n}\left[\hat{\xi}_{j, n}^{2}+(n \pi)^{2}\right]^{\frac{1}{2}} \hat{\xi}_{j, n}^{-1} \cos \theta+O\left(\hat{\xi}_{j, n}^{-1}\right), \\
& I_{r e}^{2}=O(r), \\
& I_{r e}^{3}=-\frac{\rho c^{2}(n \pi)^{2}}{|\xi|^{2}} r \cos \hat{\xi}_{j, n} \cos \theta+O\left(\hat{\xi}_{j, n}^{-1}\right), \\
& I_{r e}^{4}=-\frac{\rho c^{2}(n \pi)^{2}}{|\xi|^{2}} r \cos \hat{\xi}_{j, n} \cos \theta+O\left(\hat{\xi}_{j, n}^{-1}\right), \\
& I_{i m}^{1}=-m c^{2} \cos \hat{\xi}_{j, n}\left[\hat{\xi}_{j, n}^{2}+(n \pi)^{2}\right]^{\frac{1}{2}} \hat{\xi}_{j, n}^{-1} \sin \theta+O(r)+ \\
& I_{i m}^{2}=O(r), \quad c^{2}\left\{-\left[\hat{\xi}_{j, n}^{2}+(n \pi)^{2}\right]+k\right\} \sin \hat{\xi}_{j, n} \cosh (r \cos \theta) \cos (r \sin \theta), \\
& I_{i m}^{3}=O\left(\hat{\xi}_{j, n}^{-1}\right) .
\end{aligned}
$$

Using the fact that $\frac{1}{|\xi|^{2}}=\frac{1}{\hat{\xi}_{j, n}^{2}}-\frac{2 r \sin \theta}{|\xi|^{2} \hat{\xi}_{j, n}}-\frac{r^{2}}{|\xi|^{2} \hat{\xi}_{j, n}^{2}}$, we have

$$
\begin{gathered}
I_{i m}^{4}=\rho c^{2}\left[\hat{\xi}_{j, n}+\frac{(n \pi)^{2}}{\hat{\xi}_{j, n}}\right] \cos \hat{\xi}_{j, n} \cosh (r \cos \theta) \cos (r \sin \theta) \\
-\frac{2 \rho c^{2}(n \pi)^{2}}{|\xi|^{2}} r \cos \hat{\xi}_{j, n} \sin \theta+O\left(\hat{\xi}_{j, n}^{-1}\right) .
\end{gathered}
$$

Using (2.6), (2.7), (2.13)-(2.15) and the above results, we thus obtain the asymptotic relationship as follows

$$
\begin{aligned}
|f(\xi)| \sim m c^{2}\left[\hat{\xi}_{j, n}^{2}+(n \pi)^{2}\right]^{\frac{1}{2}} \hat{\xi}_{j, n}^{-1}+\rho c^{2} \frac{(n \pi)^{2}}{|\xi|^{2}} r \\
\text { for } \hat{\xi}_{j, n}^{2}+(n \pi)^{2} \text { sufficiently large. }
\end{aligned}
$$

Comparing (2.17) and (2.19), we know that there is a $\lambda^{\star}$ independent of the parameter $d$ and then there is a constant $\alpha^{\star}$ depending on $m, c, k, \rho$ and $\lambda^{\star}$ such that if $d \leq \alpha^{\star}$ and $\left|\hat{\lambda}_{j, n}\right| \geq \lambda^{\star}$, then $|f(\xi)|>|g(\xi)|$ for every $\xi \in \gamma_{j}^{\xi}$. By Rouche's theorem we know that the equation $f(\xi)+g(\xi)=0$ has a solution $\xi_{j, n}$ 
inside $\gamma_{j}^{\xi}$ because $f(\xi)=0$ has at least one solution inside $\gamma_{j}^{\xi}$. Therefore $(2.10)$ and (2.11) follow from (2.16) and (2.18) and theorem 1 holds. Q.E.D.

Remark 2.2: From the preceding proof it follows that if we choose $r=$ $\left[\hat{\xi}_{j, n}^{2}+(n \pi)^{2}\right]^{-\tau} \hat{\xi}_{j, n}^{-1}$ for any $\tau<\frac{1}{2}$, then there is a constant $\lambda^{\star}$ such that $(2.5)$ has a solution $\lambda_{j, n}$ in the neighborhood of $\hat{\lambda}_{j, n}$ for any $\left|\hat{\lambda}_{j, n}\right| \geq \lambda^{\star}$, that is

$$
\left|\lambda_{j, n}-\hat{\lambda}_{j, n}\right| \leq C\left[\hat{\xi}_{j, n}^{2}+(n \pi)^{2}\right]^{-\tau-\frac{1}{2}}
$$

Furthermore if we define $\xi_{j, n}$ as in theorem 1, then we have

$$
\left|\xi_{j, n}-\hat{\xi}_{j, n} i\right| \leq C\left[\hat{\xi}_{j, n}^{2}+(n \pi)^{2}\right]^{-\tau} \hat{\xi}_{j, n}^{-1}
$$

Theorem 2. Let $\lambda_{j, n}$ be the same as in theorem 1. Then there is a constant $\lambda^{\star}>0$ such that if $j^{2}+n^{2} \geq \lambda^{\star}$, then

$$
\operatorname{Re}\left(\lambda_{j, n}\right) \leq-C\left[j^{2}+n^{2}\right]^{-1}
$$

Proof. Let $\vec{\psi}_{j, n}=\left(\psi_{j, n}^{(1)}, \psi_{j, n}^{(2)}, \psi_{j, n}^{(3)}, \psi_{j, n}^{(4)}\right)$ denote an eigenfunction of $\mathcal{A}$ corresponding to the eigenvalue $\lambda_{j, n}$. From the earlier discussion of this section and from (2.2), we can specially choose

$$
\begin{array}{ll}
\psi_{j, n}^{(1)}=\cosh \left(\xi_{j, n} x\right) \cos (n \pi y), & \psi_{j, n}^{(2)}=\lambda_{j, n} \psi_{j, n}^{(1)}, \\
\psi_{j, n}^{(4)}=\xi_{j, n} \sinh \xi_{j, n} \cos (n \pi y), & \psi_{j, n}^{(3)}=\frac{\xi_{j, n}}{\lambda_{j, n}} \sinh \xi_{j, n} \cos (n \pi y) .
\end{array}
$$

Let $\xi_{j, n}=a+b i$. From theorem 1 we know that $|a| \sim\left[\hat{\xi}_{j, n}^{2}+(n \pi)^{2}\right]^{-\frac{1}{2}} \hat{\xi}_{j, n}^{-1}$ and $|b| \sim\left[\hat{\xi}_{j, n}^{2}+(n \pi)^{2}\right]^{\frac{1}{2}}$. Thus a direct calculation shows that

$$
\begin{aligned}
\int_{0}^{1}\left|\cosh \left(\xi_{j, n} x\right)\right|^{2} d x & =\int_{0}^{1}\left[\cosh ^{2}(a x) \cos ^{2}(b x)+\sinh ^{2}(a x) \sin ^{2}(b x)\right] d x \\
& =\frac{1}{2}\left[\frac{\sinh (2 a)}{2 a}+\frac{\sin (2 b)}{2 b}\right] \sim \frac{1}{2}, \\
\int_{0}^{1}\left|\sinh \left(\xi_{j, n} x\right)\right|^{2} d x & =\int_{0}^{1}\left[\sinh ^{2}(a x) \cos ^{2}(b x)+\cosh ^{2}(a x) \sin ^{2}(b x)\right] d x \\
& =\frac{1}{2}\left[\frac{\sinh (2 a)}{2 a}-\frac{\sin (2 b)}{2 b}\right] \sim \frac{1}{2}
\end{aligned}
$$


when $\hat{\xi}_{j, n}^{2}+(n \pi)^{2}$ is sufficiently large. From $(2.5)$, lemma 1 and theorem 1 we have $0<C_{1} \leq\left|\xi_{j, n} \sinh \xi_{j, n}\right| \leq C_{2}$ uniformly when $\hat{\xi}_{j, n}^{2}+(n \pi)^{2}$ is sufficiently large. Therefore from the definition (1.4) we have, when $\hat{\xi}_{j, n}^{2}+(n \pi)^{2}$ is sufficiently large,

$$
\left\|\vec{\psi}_{j, n}\right\|_{\mathcal{H}}^{2}=O\left(\left|\lambda_{j, n}\right|^{2}\right) \leq C\left|\lambda_{j, n}\right|^{2}\left\|\psi_{j, n}^{(4)}\right\|_{L^{2}(0,1)}^{2}
$$

From theorem 1 and lemma 1 we know that $\left|\lambda_{j, n}\right|^{2} \sim j^{2}+n^{2}$ for sufficiently large $j^{2}+n^{2}$. Thus (2.20) follows from (1.7) and (2.22). Q.E.D.

We have shown how a subsequence $\lambda_{j, n}$ of all eigenvalues of $\mathcal{A}$ approaches the imaginary axis when $\left|\operatorname{Im}\left(\lambda_{j, n}\right)\right|$ goes to infinity. We have also proved that the optimal rate at which $\operatorname{Re}\left(\lambda_{j, n}\right)$ approaches to the imaginary axis is $\left[j^{2}+n^{2}\right]^{-1}$. We shall show that $\pm \lambda_{j, n}$ are the only subsequences of the eigenvalues whose imaginary part goes to $\pm \infty$ in the spectrum $\sigma(\mathcal{A})$ of $\mathcal{A}$ for small positive $d$ (we actually believe this is true for any $d$ ). For the rest of this section, we show that all eigenvalues of $\mathcal{A}$ in any bounded set are bounded away from and lie to the left of the imaginary axis.

Theorem 3. Let $\lambda^{\star}>0$ be any fixed number. If $k>0$, then there is a constant $\hat{\lambda}^{\star}>0$ such that

$$
\operatorname{Re}(\lambda) \leq-\hat{\lambda}^{\star}
$$

for any eigenvalue $\lambda$ of $\mathcal{A}$ with $|\lambda| \leq \lambda^{\star}$.

Proof. This follows from the simple argument and the following lemma 2. Q.E.D.

Define $\Sigma=\left\{\lambda ; \quad m \lambda^{2}+d \lambda+k=0\right\}$. The following lemma was implicitly and partially obtained by Beale[1] who showed that the span of all generalized eigenfunctions for three dimensional model is dense in the corresponding Hilbert space. 
Lemma 2. The spectrum $\sigma(\mathcal{A})$ of $\mathcal{A}$ contains only discrete eigenvalues plus the possible accumulation points in $\Sigma$.

Proof. Without loss of generality, we may take $\lambda \neq 0$. Assume that $\lambda \notin \Sigma$. Consider the equation $(\lambda-\mathcal{A}) \vec{u})=\vec{f}$ for any $\vec{f}=\left(f^{(1)}, f^{(2)}, f^{(3)}, f^{(4)}\right) \in \mathcal{H}$. Let $\vec{u}=\left(u^{(1)}, u^{(2)}, u^{(3)}, u^{(4)}\right)$. Then we have

$$
\left\{\begin{array}{l}
-c^{2} \Delta u^{(1)}+\lambda^{2} u^{(1)}=\lambda f^{(1)}+f^{(2)} \quad \text { in } \Omega \\
\left(m \lambda^{2}+d \lambda+k\right) \frac{\partial u^{(1)}}{\partial x}+\rho \lambda^{2} u^{(1)}=\rho \lambda f^{(1)}-k f^{(3)}+m \lambda f^{(4)} \\
\frac{\partial u^{(1)}}{\partial \nu}=0 \quad \text { on } \Gamma_{1}
\end{array}\right.
$$

and

$$
u^{(2)}=\lambda u^{(1)}-f^{(1)} ; \quad u^{(4)}=\frac{\partial u^{(1)}}{\partial x} ; \quad u^{(3)}=\frac{u^{(4)}+f^{(3)}}{\lambda} .
$$

Since $m \lambda^{2}+d \lambda+k \neq 0$ and $\lambda \neq 0$ are fixed, it is well-known that mixed RobinNeumann problem (2.24) allows a unique solution $u^{(1)} \in H^{\frac{3}{2}}(\Omega)$ if the corresponding homogeneous equations of (2.24) have only the zero solution. Moreover

$$
\left\|u^{(1)}\right\|_{H^{\frac{3}{2}}(\Omega)} \leq C(\lambda)\left[\left\|f^{(1)}\right\|_{H^{\frac{1}{2}(\Omega)}}+\left\|f^{(2)}\right\|_{L^{2}(\Omega)}+\left\|f^{(3)}\right\|_{L^{2}(0,1)}+\left\|f^{(4)}\right\|_{\left.L^{2}(0,1)\right] .}(2.26)\right.
$$

Thus by the trace imbedding theorem we have $\left\|\left.\frac{\partial u^{(1)}}{\partial x}\right|_{x=1}\right\|_{L^{2}(0,1)} \leq C\left\|u^{(1)}\right\|_{H^{\frac{3}{2}}(\Omega)}$. Therefore if $\lambda$ is not an eigenvalue of $\mathcal{A}$, then $\lambda-\mathcal{A}$ is surjective and $(\lambda-\mathcal{A})^{-1}$ is a bounded operator, i.e. $\lambda$ belongs to the resolvent set of $\mathcal{A}$.

Suppose that there exists a sequence $\lambda_{j}$ of the isolated eigenvalues of the operator $\mathcal{A}$ such that $\lim _{j \rightarrow \infty} \lambda_{j}=\hat{\lambda} \notin \Sigma$. Obviously $\hat{\lambda} \neq 0$ because $\frac{1}{\lambda_{j}}$ is an eigenvalue of $\mathcal{A}^{-1}$ and $\mathcal{A}^{-1}$ is bounded. From the Riesz's theorem (cf. Dunford \& Schwartz[2]) we know that there is a sequence of eigenfunctions, $\vec{u}_{j}=\left(u_{j}^{(1)}, u_{j}^{(2)}, u_{j}^{(3)}, u_{j}^{(4)}\right)$ corresponding to the eigenvalue $\lambda_{j}$ of $\mathcal{A}$ such that 
$\left\|\vec{u}_{j}\right\|_{\mathcal{H}}=1$ for all $j$ and

$$
\left\|\vec{u}_{j_{1}}-\vec{u}_{j_{2}}\right\|_{\mathcal{H}} \geq \frac{1}{2} \quad \text { if } j_{1} \neq j_{2}
$$

But we know that $u_{j}^{(1)}$, passing to subsequence if necessary, weakly converges to $\hat{u}^{(1)}$ in $H^{1}(\Omega)$ because the set $\left\{u_{j}^{(1)}\right\}$ is bounded in $H^{1}(\Omega)$. Thus $u_{j}^{(1)}$ converges to $\hat{u}^{(1)}$ in $L^{2}(\Omega)$. Let $p(\lambda)=\frac{\rho \lambda^{2}}{m \lambda^{2}+d \lambda+k}$. From $(2.1)$ we have

$$
\begin{aligned}
\left\|u_{j_{1}}^{(1)}-u_{j_{2}}^{(1)}\right\|_{H^{\frac{3}{2}}(\Omega)} & \leq C\left[\left\|\lambda_{j_{1}} u_{j_{1}}^{(1)}-\lambda_{j_{2}} u_{j_{2}}^{(1)}\right\|_{L^{2}(\Omega)}\right. \\
& +\left\|\left.\left[p\left(\lambda_{j_{1}}\right) u_{j_{1}}^{(1)}-p\left(\lambda_{j_{2}}\right) u_{j_{2}}^{(1)}\right]\right|_{x=1}\right\|_{L^{2}(0,1)} .
\end{aligned}
$$

It follows from $(2.28)$ that $u_{j}^{(1)}$ converges to $\hat{u}^{(1)}$ in $H^{\frac{3}{2}}(\Omega)$. Thus it follows from (2.2) that $\vec{u}_{j}$ converges in $\mathcal{H}$. This is a contradiction to (2.27). $\quad$ Q.E.D.

Remark 2.3: $\lambda-\mathcal{A}$ for $\lambda \in \Sigma$ is called singular (cf. Beale[1]), i.e. there is a sequence $\vec{u}_{j} \in \mathcal{D}(\mathcal{A})$ with no convergent subsequence such that $\left\|\vec{u}_{j}\right\|_{\mathcal{H}}=1$ and $(\lambda-\mathcal{A}) \vec{u}_{j} \rightarrow 0$. In the case of our model the range $R(\lambda-\mathcal{A})$ is not closed but dense in $\mathcal{H}$.

\section{The Expansion.}

In this section we shall use perturbation theory (cf. Kato[5]) and a part of Beale[1]'s results (In fact, similar results) to show that provided the parameter $d$ is small, any element of the Hilbert space $\mathcal{H}$ can be decomposed as the sum of a) an infinite linear combination of set $\left\{\vec{\psi}_{j, n} ; \vec{\psi}_{j, n}\right\}_{j^{2}+n^{2} \geq \lambda^{\star}}$ for some $\lambda^{\star}>$ 0 ; and b) the projection on the closure in $\mathcal{H}$ of the span of all generalized eigenfunctions of the corresponding eigenvalues of $\mathcal{A}$, bounded in absolute value by $\lambda^{\star}$ (if necessary, $\lambda^{\star}$ can be changed). We even believe that this is true for any positive constant $d$ but can not prove it. This result is much stronger than the statement that the span of all generalized eigenfunctions of $\mathcal{A}$ is dense in the Hilbert space $\mathcal{H}$. We shall use this result to study the energy decay rates of problem (1.1). 
In the following discussion, all closed simple curves are positively oriented. Let $\gamma$ be a closed simple curve in the resolvent set of $\mathcal{A}$ such that it encloses the set $\Sigma$. We define a projection operator $\mathcal{P}_{\gamma}$ as follows:

$$
\mathcal{P}_{\gamma}=\frac{1}{2 \pi i} \int_{\gamma}(\lambda-\mathcal{A})^{-1} d \lambda .
$$

Then $R\left(\mathcal{P}_{\gamma}\right) \subset \mathcal{D}(\mathcal{A})$ and $\mathcal{P}_{\gamma}$ commutes with $\mathcal{A}$. If we set $Q=I-\mathcal{P}_{\gamma}$, then $\mathcal{H}=\mathcal{P}_{\gamma} \mathcal{H} \oplus Q \mathcal{H}$. We denote by $\operatorname{sp}(T)$ the closure of the span of all generalized eigenfunctions $\left\{\vec{u} \in \mathcal{H} ; \quad(\lambda-T)^{j} \vec{u}=\overrightarrow{0} \quad\right.$ for some $\lambda$ and a positive integer $\left.j\right\}$ for any linear operator $T$ from $\mathcal{D}(T) \subset \mathcal{H}$ to $\mathcal{H}$.

Lemma 3(cf. Beale[1]). If $d>0$, then $\operatorname{sp}\left(\mathcal{A P}_{\gamma}\right)=\mathcal{P}_{\gamma} \mathcal{H}$

Proof. The argument is similar to the proofs of several lemmas and theorems in Beale[1]. To be self contained, we here just give a draft of the proof but readers can refer Beale[1] for details.

Without loss of generality, we assume that $\Sigma$ consists of two different numbers $\tilde{\lambda}_{1}$ and $\tilde{\lambda}_{2}$ (this is true for $k>0$ and small data $d$ ). Choosing two points on the curve $\gamma$, we connect those two points by a curve in the resolvent set of $\mathcal{A}$ in such way that we decompose the closed simple curve $\gamma$ into two closed simple curves $\tilde{\gamma}_{l}, l=1,2$ such that $\tilde{\gamma}_{l}$ encloses $\tilde{\lambda}_{l}$ respectively. Let $\mathcal{P}_{\tilde{\gamma}_{l}}, \quad l=1,2$ be defined as (3.1) on $\tilde{\gamma}_{l}, \quad l=1,2$ respectively. Then once again the $\mathcal{P}_{\tilde{\gamma}_{l}}, l=1,2$ commute with $\mathcal{A}$ and $\mathcal{P}_{\gamma}=\mathcal{P}_{\tilde{\gamma}_{1}}+\mathcal{P}_{\tilde{\gamma}_{2}}$. It follows from the proof of lemma 4.3 of Beale[1] that for $l=1,2,\left(\tilde{\lambda}_{l}-\mathcal{A} \mathcal{P}_{\tilde{\gamma}_{l}}\right)^{2}$ is a Hilbert-Schmidt operator (see Dunford \& Schwartz[2], Sec. XI.6 for the definition and also see Kato[5]) on $\mathcal{P}_{\tilde{\gamma}_{l}} \mathcal{H}$ and $\tilde{\lambda}_{l}-\mathcal{A} \mathcal{P}_{\tilde{\gamma}_{l}}$ is also a compact operator on $\mathcal{P}_{\tilde{\gamma}_{l}} \mathcal{H}$ respectively. Since the following problem

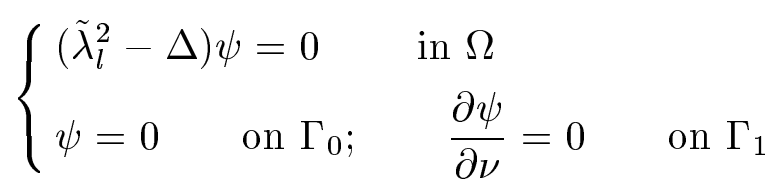


has only the zero solution because for $l=1,2, \tilde{\lambda}_{l}$ is not a purely imaginary number, the following result follows from the argument of lemma 4.5 of Beale[1].

Let $\psi_{j}$ be a sequence in $H^{1}(\Omega)$ such that

$$
\left\{\begin{array}{l}
\left(\lambda_{j}^{2}-\Delta\right) \psi_{j}=h_{j} \quad \text { in } \Omega \\
\frac{\partial \psi_{j}}{\partial \nu}+p\left(\lambda_{j}\right) \psi_{j}=e_{j} \quad \text { on } \Gamma_{0} \\
\frac{\partial \psi_{j}}{\partial \nu}=0 \quad \text { on } \Gamma_{1}
\end{array}\right.
$$

where $p(\lambda)$ is the same as in (2.28), $\lambda_{j}=\tilde{\lambda}_{1}+\epsilon_{j} \eta$ with $|\eta|=1$ and real $\epsilon_{j} \rightarrow 0$ as $j \rightarrow \infty$. If $h_{j}$ and $e_{j}$ is a bounded sequence in $L^{2}(\Omega)$ and $L^{2}(0,1)$ respectively, then $\psi_{j}$ is a bounded sequence in $H^{1}(\Omega)$ unless $\eta$ is one of at most two exceptional values (which make the imaginary parts of $\frac{\tilde{\lambda}_{1}^{2}}{\eta\left(\tilde{\lambda}_{1}-\tilde{\lambda}_{2}\right)}$ zero).

The same result holds for $\tilde{\lambda}_{2}$. With these results, we claim that lemma 3 holds via the proof of theorem 4.4 of Beale[1]. $\quad$ Q.E.D.

Remark 3.1: From the above proof it follows that we have that the order of the pole of each fixed eigenvalue $\lambda \notin \Sigma$ is finite, i.e. $\inf \left\{j ; N\left((\lambda-\mathcal{A})^{j}\right)=\right.$ $\left.N\left((\lambda-\mathcal{A})^{j+1}\right)\right\}<\infty$ (cf. Dunford \& Schwartz[2]). Also for each eigenvalue $\lambda \notin \Sigma, \lambda-\mathcal{A}$ is a Fredholm operator of index zero (cf. theorem 3.2 of Beale[1]).

$$
\begin{aligned}
& \text { Let } \overrightarrow{\hat{\psi}}_{j, n}=\left(\hat{\psi}_{j, n}^{(1)}, \hat{\psi}_{j, n}^{(2)}, \hat{\psi}_{j, n}^{(3)}, \hat{\psi}_{j, n}^{(4)}\right) \text { where } \\
& \hat{\psi}_{j, n}^{(1)}=\cosh \left(i \hat{\xi}_{j, n} x\right) \cos (n \pi y), \quad \hat{\psi}_{j, n}^{(2)}=\hat{\lambda}_{j, n} \hat{\psi}_{j, n}^{(1)}, \\
& \hat{\psi}_{j, n}^{(4)}=i \hat{\xi}_{j, n} \sinh \left(i \hat{\xi}_{j, n}\right) \cos (n \pi y), \quad \hat{\psi}_{j, n}^{(3)}=\frac{i \hat{\xi}_{j, n}}{\hat{\lambda}_{j, n}} \sinh \left(i \hat{\xi}_{j, n}\right) \cos (n \pi y)
\end{aligned}
$$

in which $\hat{\xi}_{j, n}$ and $\hat{\lambda}_{j, n}$ have the same meanings as in $\S 2$. Then in the case $d=0$, $\overrightarrow{\hat{\psi}}_{j, n}$ is an eigenfunction corresponding to the eigenvalue $\hat{\lambda}_{j, n}$ of the operator $\mathcal{A}$.

Lemma 4. The constants $\lambda^{\star}$ and $\alpha^{\star}$ of theorem 1 can be chosen such that if 
$d \leq \alpha^{\star}$, then

$$
\sum_{\left|\hat{\lambda}_{j, n}\right| \geq \lambda^{\star}}\left\|\frac{\vec{\psi}_{j, n}}{\left\|\vec{\psi}_{j, n}\right\|_{\mathcal{H}}}-\frac{\overrightarrow{\hat{\psi}}_{j, n}}{\left\|\overrightarrow{\hat{\psi}}_{j, n}\right\|_{\mathcal{H}}}\right\|_{\mathcal{H}}^{2}<\infty
$$

where $\vec{\psi}_{j, n}$ are the same as in theorem 2.

Proof. It follows from (1.4), (2.21), (3.2) and theorem 1 that we have

$$
\begin{aligned}
\left\|\vec{\psi}_{j, n}-\overrightarrow{\hat{\psi}}_{j, n}\right\|_{\mathcal{H}}^{2} & \leq C\left|\hat{\lambda}_{j, n}\right|^{2}\left[\left|\lambda_{j, n}-\hat{\lambda}_{j, n}\right|^{2}+\left|\xi_{j, n}-i \hat{\xi}_{j, n}\right|^{2}\right] \\
& \leq C\left[\frac{1}{\hat{\xi}_{j, n}^{2}+(n \pi)^{2}}+\frac{1}{\left|\hat{\xi}_{j, n}\right|^{2}}\right]
\end{aligned}
$$

when $\hat{\xi}_{j, n}^{2}+(n \pi)^{2}$ is sufficiently large and $d \leq \alpha^{\star}$. Since $\left\|\overrightarrow{\hat{\psi}}_{j, n}\right\|_{\mathcal{H}}^{2} \sim\left\|\vec{\psi}_{j, n}\right\|_{\mathcal{H}}^{2}=$ $O\left(\left|\hat{\lambda}_{j, n}\right|^{2}\right)$ by (2.22) and theorem 1, from (3.4) we have

$$
\begin{aligned}
& \sum_{\left|\hat{\lambda}_{j, n}\right| \geq \lambda^{\star}}\left\|\frac{\vec{\psi}_{j, n}}{\left\|\vec{\psi}_{j, n}\right\|_{\mathcal{H}}}-\frac{\overrightarrow{\hat{\psi}}_{j, n}}{\left\|\overrightarrow{\hat{\psi}}_{j, n}\right\|_{\mathcal{H}}}\right\|_{\mathcal{H}}^{2} \\
& \leq C \sum_{\left|\hat{\lambda}_{j, n}\right| \geq \lambda^{\star}}\left[\frac{\left|\hat{\lambda}_{j, n}-\lambda_{j, n}\right|}{\left|\lambda_{j, n}\right|^{2}\left|\hat{\lambda}_{j, n}\right|^{2}}\left\|\vec{\psi}_{j, n}\right\|_{\mathcal{H}}^{2}+\frac{1}{\left|\hat{\lambda}_{j, n}\right|^{2}}\left\|\vec{\psi}_{j, n}-\overrightarrow{\hat{\psi}}_{j, n}\right\|_{\mathcal{H}}^{2}\right] \\
& \leq C\left\{\sum_{\left|\hat{\lambda}_{j, n}\right| \geq \lambda^{\star}} \frac{1}{\left[\hat{\xi}_{j, n}^{2}+(n \pi)^{2}\right]^{2}}+\sum_{\left|\hat{\lambda}_{j, n}\right| \geq \lambda^{\star}} \frac{1}{\hat{\xi}_{j, n}^{2}\left[\hat{\xi}_{j, n}^{2}+(n \pi)^{2}\right]}\right\} .
\end{aligned}
$$

Obviously we have $\sum_{\left|\hat{\lambda}_{j, n}\right| \geq \lambda^{\star}} \frac{1}{\left[\hat{\xi}_{j, n}^{2}+(n \pi)^{2}\right]^{2}}<\infty$ by lemma 1. From lemma 1 we also have that if $\lambda^{\star}$ is suitable large, then

$$
\begin{aligned}
& \sum_{\left|\hat{\lambda}_{j, n}\right| \geq \lambda^{\star}} \frac{1}{\hat{\xi}_{j, n}^{2}\left[\hat{\xi}_{j, n}^{2}+(n \pi)^{2}\right]} \leq C \sum_{j^{2}+n^{2} \geq \lambda_{1}^{\star}} \frac{1}{j^{2}\left(j^{2}+n^{2}\right)} \\
& \quad \leq C \int_{1}^{+\infty} \int_{1}^{+\infty} \frac{1}{x^{2}\left(x^{2}+y^{2}\right)} d y d x \leq\left. C \int_{1}^{+\infty} \frac{1}{x^{3}} \tan \left(\frac{y}{x}\right)\right|_{1} ^{+\infty} d x<+\infty
\end{aligned}
$$

where $\lambda_{1}^{\star}$ is a suitable number such that $\left|\hat{\lambda}_{j, n}\right| \geq \lambda^{\star}$ if and only if $j^{2}+n^{2} \geq \lambda_{1}^{\star}$. Thus lemma 4 holds and (3.3) follows from (3.5) and (3.6). Q.E.D.

Using the idea of a Bary basis (cf. Kato[5], Chap.5, §2.3), we now claim the following "Fourier" expansion theorem. 
Theorem 4. There are a closed simple curve $\gamma^{\star}$ in the resolvent set of $\mathcal{A}$ and positive constants $\lambda^{\star}, \alpha^{\star}\left(\lambda^{\star}, \gamma^{\star}\right)$ such that if $d \leq \alpha^{\star}$, then for any given $\vec{\psi} \in \mathcal{H}$, there are two unique sequences $\left\{\eta_{j, n}^{(1)} ; \eta_{j, n}^{(2)}\right\}_{j^{2}+n^{2} \geq \lambda^{\star}}$ and a vector function $\vec{\varphi} \in \mathcal{H}$ such that

$$
\vec{\psi}=\mathcal{P}_{\gamma^{\star}} \vec{\varphi}+\sum_{j^{2}+n^{2} \geq \lambda^{\star}}\left[\eta_{j, n}^{(1)} \frac{\vec{\psi}_{j, n}}{\left\|\vec{\psi}_{j, n}\right\|_{\mathcal{H}}}+\eta_{j, n}^{(2)} \frac{\overrightarrow{\vec{\psi}}_{j, n}}{\left\|\vec{\psi}_{j, n}\right\|_{\mathcal{H}}}\right] .
$$

Moreover there are positive constants $C_{1}$ and $C_{2}$ such that

$$
C_{1}\|\vec{\psi}\|_{\mathcal{H}}^{2} \leq\|\vec{\varphi}\|_{\mathcal{H}}^{2}+\sum_{j^{2}+n^{2} \geq \lambda^{\star}}\left[\left|\eta_{j, n}^{(1)}\right|^{2}+\left|\eta_{j, n}^{(2)}\right|^{2}\right] \leq C_{2}\|\vec{\psi}\|_{\mathcal{H}}^{2}
$$

Proof. From lemma 4 we know that there exist constants $\lambda^{\star}>0$ and $\alpha^{\star}\left(\lambda^{\star}\right)$ such that if $d \leq \alpha^{\star}$, then

$$
\sum_{\left|\hat{\lambda}_{j, n}\right| \geq \lambda^{\star}}\left\|\frac{\vec{\psi}_{j, n}}{\left\|\vec{\psi}_{j, n}\right\|_{\mathcal{H}}}-\frac{\overrightarrow{\hat{\psi}}_{j, n}}{\left\|\overrightarrow{\hat{\psi}}_{j, n}\right\|_{\mathcal{H}}}\right\|_{\mathcal{H}}^{2}<\frac{1}{4}
$$

Let $\mathcal{A}_{0}$ denote $\mathcal{A}$ for the case $d=0$. Then by the argument of lemma 1 we can also choose $\lambda^{\star}$ sufficiently large such that all of the eigenvalues of $\mathcal{A}_{0}$ whose absolute values are greater than or equal to $\lambda^{\star}$ are given by $\hat{\lambda}_{j, n}$ and $\overline{\hat{\lambda}}_{j, n}$. Let $\gamma^{\star}$ be a closed simple curve in the resolvent set of $\mathcal{A}_{0}$ such that $\gamma^{\star}$ encloses all of $\Sigma$ for $d \leq \alpha^{\star}$ and all of eigenvalues of $\mathcal{A}_{0}$ whose absolute values are less than $\lambda^{\star}$. Let

$$
\mathcal{P}_{\gamma^{\star}}^{0}=\frac{1}{2 \pi i} \int_{\gamma^{\star}}\left(\lambda-\mathcal{A}_{0}\right)^{-1} d \lambda
$$

Since we know from proposition 1 that $\mathcal{A}_{0}$ is a skew-adjoint operator, we have that $\left\|\left(\lambda-\mathcal{A}_{0}\right)^{-1}\right\|=\sup _{\hat{\lambda} \in \sigma\left(\mathcal{A}_{0}\right)}|\lambda-\hat{\lambda}|^{-1}$ for any fixed $\lambda \in \gamma^{\star}$. It is easy to show that $\left\|\mathcal{A}-\mathcal{A}_{0}\right\| \leq \frac{d}{m}$ by (1.4). Thus if $d<m \cdot \inf _{\lambda \in \gamma^{\star}} \inf _{\hat{\lambda} \in \sigma\left(\mathcal{A}_{0}\right)}|\lambda-\hat{\lambda}|$, remembering that $\inf _{\lambda \in \gamma^{\star}} \inf _{\hat{\lambda} \in \sigma\left(\mathcal{A}_{0}\right)}|\lambda-\hat{\lambda}|>0$ is provided by lemma 2 and the choice of $\gamma^{\star}$, then 
$(\lambda-\mathcal{A})^{-1}$ for $\lambda \in \gamma^{\star}$ exists and moreover

$$
\begin{aligned}
\left\|(\lambda-\mathcal{A})^{-1}\right\| & \leq\left\|\left(\lambda-\mathcal{A}_{0}\right)^{-1}\right\| \cdot\left\|\left[I-\left(\lambda-\mathcal{A}_{0}\right)^{-1}\left(\mathcal{A}-\mathcal{A}_{0}\right)\right]^{-1}\right\| \\
& \leq \frac{\left\|\left(\lambda-\mathcal{A}_{0}\right)^{-1}\right\|}{1-\frac{d}{m} \sup _{\hat{\lambda} \in \sigma\left(\mathcal{A}_{0}\right)}|\lambda-\hat{\lambda}|^{-1}} \quad \forall \lambda \in \gamma^{\star} .
\end{aligned}
$$

That means that $\gamma^{\star}$ also lies in the resolvent set of $\mathcal{A}$ for $d<m \cdot \inf _{\lambda \in \gamma^{\star}} \inf _{\hat{\lambda} \in \sigma\left(\mathcal{A}_{0}\right)} \mid \lambda-$ $\hat{\lambda} \mid$. Thus $\mathcal{P}_{\gamma^{\star}}$ in (3.1) is well-defined. We also have

$$
\left\|(\lambda-\mathcal{A})^{-1}-\left(\lambda-\mathcal{A}_{0}\right)^{-1}\right\| \leq \frac{d}{m}\left\|(\lambda-\mathcal{A})^{-1}\right\| \cdot\left\|\left(\lambda-\mathcal{A}_{0}\right)^{-1}\right\| \quad \forall \lambda \in \gamma^{\star}
$$

Since $\left\|\left(\lambda-\mathcal{A}_{0}\right)^{-1}\right\|$ is uniformly bounded on $\gamma^{\star}$, it follows from (3.1) and (3.10)(3.12) that there exists a constant $\alpha_{1}^{\star}\left(\gamma^{\star}\right)>0$ such that

$$
\left\|\mathcal{P}_{\gamma^{\star}}^{0}-\mathcal{P}_{\gamma^{\star}}\right\|^{2}<\frac{1}{2} \quad \text { if } d \leq \alpha_{1}^{\star}\left(\gamma^{\star}\right)
$$

Since $\overrightarrow{\hat{\psi}}_{j, n}$ and $\overrightarrow{\hat{\psi}}_{j_{1}, n_{1}}$ for $\hat{\lambda}_{j, n}=\hat{\lambda}_{j_{1}, n_{1}}$ but $n \neq n_{1}$ are orthogonal, by proposition 1 it is easy to check that all of the $\overrightarrow{\hat{\psi}}_{j, n}$ and $\overline{\hat{\hat{\psi}}}_{j, n}$ for $\left|\hat{\lambda}_{j, n}\right| \geq \lambda^{\star}$ are orthogonal. Thus from proposition 1 and lemma 2 we know that for any $\vec{\psi} \in \mathcal{H}$, there are two unique sequences $\left\{\eta_{j, n}^{(1)} ; \eta_{j, n}^{(2)}\right\}_{\left|\hat{\lambda}_{j, n}\right| \geq \lambda^{\star}}$ such that

$$
\vec{\psi}=\mathcal{P}_{\gamma^{\star}}^{0} \vec{\psi}+\sum_{\left|\hat{\lambda}_{j, n}\right| \geq \lambda^{\star}}\left[\eta_{j, n}^{(1)} \frac{\overrightarrow{\hat{\psi}}_{j, n}}{\left\|\overrightarrow{\hat{\psi}}_{j, n}\right\|_{\mathcal{H}}}+\eta_{j, n}^{(2)} \frac{\overrightarrow{\hat{\hat{\psi}}}_{j, n}}{\left\|\overrightarrow{\hat{\psi}}_{j, n}\right\|_{\mathcal{H}}}\right] .
$$

Moreover we have

$$
\|\vec{\psi}\|_{\mathcal{H}}^{2}=\left\|\mathcal{P}_{\gamma^{\star}}^{0} \vec{\psi}\right\|_{\mathcal{H}}^{2}+\sum_{\left|\hat{\lambda}_{j, n}\right| \geq \lambda^{\star}}\left[\left|\eta_{j, n}^{(1)}\right|^{2}+\left|\eta_{j, n}^{(2)}\right|^{2}\right]
$$

We define a linear operator $T$ in $\mathcal{H}$ by

$$
T \vec{\psi}=\mathcal{P}_{\gamma^{\star}}\left(\mathcal{P}_{\gamma^{\star}}^{0} \vec{\psi}\right)+\sum_{\left|\hat{\lambda}_{j, n}\right| \geq \lambda^{\star}}\left[\eta_{j, n}^{(1)} \frac{\vec{\psi}_{j, n}}{\left\|\vec{\psi}_{j, n}\right\|_{\mathcal{H}}}+\eta_{j, n}^{(2)} \frac{\overrightarrow{\vec{\psi}}_{j, n}}{\left\|\vec{\psi}_{j, n}\right\|_{\mathcal{H}}}\right]
$$


if $\vec{\psi}$ has the expansion (3.14). To see that this definition is possible, it is sufficient to prove that the series $T \vec{\psi}-\vec{\psi}=\left(\mathcal{P}_{\gamma^{\star}}-\mathcal{P}_{\gamma^{\star}}^{0}\right) \mathcal{P}_{\gamma^{\star}}^{0} \vec{\psi}+\sum_{\left|\hat{\lambda}_{j, n}\right| \geq \lambda^{\star}}\left[\eta_{j, n}^{(1)}\left(\frac{\vec{\psi}_{j, n}}{\left\|\vec{\psi}_{j, n}\right\|_{\mathcal{H}}}-\right.\right.$ $\left.\left.\frac{\overrightarrow{\hat{\psi}}_{j, n}}{\left\|\overrightarrow{\hat{\psi}}_{j, n}\right\|_{\mathcal{H}}}\right)+\eta_{j, n}^{(2)}\left(\frac{\overline{\vec{\psi}}_{j, n}}{\left\|\vec{\psi}_{j, n}\right\|_{\mathcal{H}}}-\frac{\overline{\hat{\hat{\psi}}}_{j, n}}{\left\|\overrightarrow{\hat{\psi}}_{j, n}\right\|_{\mathcal{H}}}\right)\right]$ converges absolutely. This is proved by means of the following Schwarz inequality

$$
\begin{aligned}
\|T \vec{\psi}-\vec{\psi}\|_{\mathcal{H}}^{2} \leq\left\{\left\|\mathcal{P}_{\gamma^{\star}}^{0} \vec{\psi}\right\|_{\mathcal{H}}^{2}+\sum_{\left|\hat{\lambda}_{j, n}\right| \geq \lambda^{\star}}\left[\left|\eta_{j, n}^{(1)}\right|^{2}+\left|\eta_{j, n}^{(2)}\right|^{2}\right]\right\} \cdot \\
\cdot\left\{\left\|\mathcal{P}_{\gamma^{\star}}^{0}-\mathcal{P}_{\gamma^{\star}}\right\|^{2}+2 \sum_{\left|\hat{\lambda}_{j, n}\right| \geq \lambda^{\star}}\left\|\frac{\vec{\psi}_{j, n}}{\left\|\vec{\psi}_{j, n}\right\|_{\mathcal{H}}}-\frac{\overrightarrow{\hat{\psi}}_{j, n}}{\left\|\overrightarrow{\hat{\psi}}_{j, n}\right\|_{\mathcal{H}}}\right\|_{\mathcal{H}}^{2}\right\} .
\end{aligned}
$$

Thus by 3.17 we have that $T$ is a bounded operator and $\|T-I\|<1$. Therefore $T^{-1}$ exists and is bounded. So for any $\vec{\psi} \in \mathcal{H}$, we take $\vec{\varphi}=\mathcal{P}_{\gamma^{\star}}^{0}\left(T^{-1} \vec{\psi}\right)$. As in the proof of lemma 4 one can change the indexing for the above series from $\left|\hat{\lambda}_{j, n}\right| \geq \lambda^{\star}$ to $j^{2}+n^{2} \geq \lambda^{\star}$ ( $\lambda^{\star}$ needs to be changed). Thus we know that theorem 4 holds and (3.8) follows from (3.14) and that $T, T^{-1}$ are bounded. Q.E.D.

\section{Energy Decay Rates.}

We first prove a lemma about how the energy of system (1.1) decays rationally. This idea can be found in Littman \& Markus[6] but there all of the eigenvalues and eigenfunctions were indexed by one parameter. Here we have

Lemma 5. Assume that $\vec{u}_{0}=\sum_{j^{2}+n^{2} \geq \lambda^{\star}}\left[\eta_{j, n}^{(1)} \frac{\vec{\psi}_{j, n}}{\left\|\vec{\psi}_{j, n}\right\|_{\mathcal{H}}}+\eta_{j, n}^{(2)} \frac{\overline{\vec{\psi}}_{j, n}}{\left\|\vec{\psi}_{j, n}\right\|_{\mathcal{H}}}\right]$ with $\left|\eta_{j, n}^{(1)}\right|$, $\left|\eta_{j, n}^{(2)}\right| \leq \frac{C}{\left(j^{2}+n^{2}\right)^{\beta}}$ where $\beta>\frac{1}{2}$. Let $\vec{u}(t) \in \mathcal{H}$ be the solution of (1.6) with respect to the initial data $\vec{u}_{0}$. Then

$$
\|\vec{u}(t)\|_{\mathcal{H}} \leq C t^{-\frac{2 \beta-1}{2}}, \quad t>0
$$


Proof. Since $\vec{\psi}_{j, n}, \vec{\psi}_{j, n}$ for $j^{2}+n^{2} \geq \lambda^{\star}$ are eigenfunctions of $\mathcal{A}$, we have

$$
\vec{u}(t)=\sum_{j^{2}+n^{2} \geq \lambda^{\star}}\left[\eta_{j, n}^{(1)} \frac{e^{\lambda_{j, n} t} \vec{\psi}_{j, n}}{\left\|\vec{\psi}_{j, n}\right\|_{\mathcal{H}}}+\eta_{j, n}^{(2)} \frac{e^{\bar{\lambda}_{j, n} t} \overline{\vec{\psi}}_{j, n}}{\left\|\vec{\psi}_{j, n}\right\|_{\mathcal{H}}}\right] .
$$

Thus from theorem 2 and 4 and the assumptions we have

$$
\begin{aligned}
\|\vec{u}(t)\|_{\mathcal{H}}^{2} & \leq C \sum_{j^{2}+n^{2} \geq \lambda^{\star}}\left[\left|\eta_{j, n}^{(1)}\right|^{2}+\left|\eta_{j, n}^{(2)}\right|^{2}\right] e^{2 R e\left(\lambda_{j, n}\right) t} \\
& \leq C \sum_{j^{2}+n^{2} \geq \lambda^{\star}} \frac{1}{\left(j^{2}+n^{2}\right)^{2 \beta}} e^{-2 C^{\prime} t /\left(j^{2}+n^{2}\right)} \\
& \leq C \iiint_{x^{2}+y^{2} \geq \lambda^{\star}} \frac{1}{\left(x^{2}+y^{2}\right)^{2 \beta}} e^{-2 C^{\prime} t /\left(x^{2}+y^{2}\right)} d x d y \\
& \leq C \int_{\sqrt{\lambda^{\star}}}^{\infty} \frac{1}{r^{4 \beta-1}} e^{-\frac{2 C^{\prime} t}{r^{2}}} d r \\
& \leq C t^{-(2 \beta-1)} \int_{0}^{\infty} e^{-\mu} \mu^{2 \beta-2} d \mu \leq C t^{-(2 \beta-1)}, \quad t>0
\end{aligned}
$$

where $C^{\prime}$ is a positive constant inherited from theorem 2. Thus (4.1) follows from (4.3). Q.E.D.

How do we convert the smoothness of $\vec{\psi} \in \mathcal{H}$ to that the coefficients $\eta_{j, n}^{(1)}$, $\eta_{j, n}^{(2)}$ in the "Fourier" expansion of $\vec{\psi}$ satisfy the assumptions of lemma 5 ? We have

Lemma 6. Assume that $\vec{\psi}=\left(\psi^{(1)}, \psi^{(2)}, \psi^{(3)}, \psi^{(4)}\right) \in \mathcal{D}(\mathcal{A})$ has the expansion in (3.7) where $\lambda^{\star}$ is assumed to be sufficiently large. If $\psi^{(1)} \in H^{3}(\Omega)$ and $\psi^{(2)} \in H^{2}(\Omega)$ with $\frac{\partial \psi^{(2)}}{\partial \nu}=0 \quad$ a.e. on $\partial \Omega$, then

$$
\begin{array}{r}
\left|\eta_{j, n}^{(1)}\right|+\left|\eta_{j, n}^{(2)}\right| \leq C\left[j^{2}+n^{2}\right]^{-1}\left[\left\|\psi^{(1)}\right\|_{H^{3}(\Omega)}+\left\|\psi^{(2)}\right\|_{H^{2}(\Omega)}+\left\|\psi^{(3)}\right\|_{L^{2}(0,1)}\right] \\
\text { for } j^{2}+n^{2} \geq \lambda^{\star} .
\end{array}
$$

Proof. We look at the dual orthogonal set of $\left\{\frac{\vec{\psi}_{j, n}}{\left\|\vec{\psi}_{j, n}\right\|_{\mathcal{H}}} ; \frac{\vec{\psi}_{j, n}}{\left\|\vec{\psi}_{j, n}\right\|_{\mathcal{H}}}\right\}_{j^{2}+n^{2} \geq \lambda^{\star}}$. Since $0=\left\langle(\lambda-\mathcal{A}) \vec{\psi}, \vec{\psi}^{\star}\right\rangle_{\mathcal{H}}=\left(\lambda-\overline{\lambda^{\dagger}}\right)\left\langle\vec{\psi}, \vec{\psi}^{\star}\right\rangle_{\mathcal{H}}$ for the eigenfunction $\vec{\psi}$ corresponding 
to the eigenvalue $\lambda$ of $\mathcal{A}$ and the eigenfunction $\vec{\psi}^{\star}$ corresponding to the eigenvalue $\lambda^{\dagger}$ of $\mathcal{A}^{\star}$ where $\mathcal{A}^{\star}$ denotes the adjoint operator, we know that the (even generalized) eigenfunction $\vec{\psi}$ of $\mathcal{A}$ and the eigenfunction $\vec{\psi}^{\star}$ of $\mathcal{A}^{\star}$ must be orthogonal when $\lambda \neq \overline{\lambda^{\dagger}}$. Since $\sigma\left(\mathcal{A}^{\star}\right)=\overline{\sigma(\mathcal{A})}=\sigma(\mathcal{A})$ and $\mathcal{A}^{\star}=\mathcal{A}-2 \mathcal{A}_{0}$ where $\mathcal{A}_{0}$ is the same as in the proof of theorem 4 , a straight calculation shows that the eigenfunction $\vec{\psi}_{j, n}^{\star}$ corresponding to the eigenvalue $\lambda_{j, n}$ of $\mathcal{A}^{\star}$ is given by

$$
\vec{\psi}^{\star}=\left(\psi_{j, n}^{(1)},-\psi_{j, n}^{(2)},-\psi_{j, n}^{(3)}, \psi_{j, n}^{(4)}\right)
$$

where $\psi_{j, n}^{(h)}, h=1,2,3,4$ are given by $(2.21)$. By lemma 3 we have that $\vec{\psi}^{\star}$ for $j^{2}+n^{2} \geq \lambda^{\star}$ is orthogonal to $\mathcal{P}_{\gamma^{\star}} \mathcal{H}$. Since $\cos (n \pi y)$ and $\cos \left(n_{1} \pi y\right)$ for $n \neq n_{1}$ are orthogonal even though $\lambda_{j, n}=\lambda_{j_{1}, n_{1}}$, from (3.7) we have

$$
\eta_{j, n}^{(1)}=\left\langle\vec{\psi}, \overline{\vec{\psi}}^{\star}{ }_{j, n}\right\rangle_{\mathcal{H}} /\left\langle\frac{\vec{\psi}_{j, n}}{\left\|\vec{\psi}_{j, n}\right\|_{\mathcal{H}}}, \overline{\vec{\psi}}^{\star}{ }_{j, n}\right\rangle_{\mathcal{H}} .
$$

By (1.4), (2.21), (4.5) and theorem 1, a direct calculation shows

$$
\begin{aligned}
\left|\left\langle\vec{\psi}_{j, n},{\overrightarrow{\psi^{\star}}}_{j, n}\right\rangle_{\mathcal{H}}\right| & =\left|-\frac{1}{2} p c^{-2} \lambda_{j, n}^{2}+\int_{\Gamma_{0}}\left[-k\left(\psi_{j, n}^{(3)}\right)^{2}+m\left(\psi_{j, n}^{(4)}\right)^{2}\right] d y\right| \\
& =O\left(\left|\lambda_{j, n}\right|^{2}\right) \geq C\left[j^{2}+n^{2}\right] \quad \text { for } j^{2}+n^{2} \geq \lambda^{\star}
\end{aligned}
$$

From the assumptions, the second equation of (2.1) and integration by parts we have

$$
\begin{aligned}
\iint_{\Omega} \nabla\left(\Delta \psi^{(1)}\right) \cdot \nabla \psi_{j, n}^{(1)} d x d y & =-\lambda_{j, n}^{2} \iint_{\Omega} \Delta \psi^{(1)} \psi_{j, n}^{(1)} d x d y+\int_{\Gamma_{0}} \Delta \psi^{(1)} \psi_{j, n}^{(4)} d y \\
= & \lambda_{j, n}^{2} \iint_{\Omega} \nabla \psi^{(1)} \cdot \nabla \psi_{j, n}^{(1)} d x d y+\frac{1}{\rho}\left(m \lambda_{j, n}^{2}+d \lambda_{j, n}+k\right) \cdot \\
& \cdot \int_{\Gamma_{0}} \psi^{(4)} \psi_{j, n}^{(4)} d y+\int_{\Gamma_{0}} \Delta \psi^{(1)} \psi_{j, n}^{(4)} d y
\end{aligned}
$$

From (4.8), (2.22), theorem 1 and the trace imbedding theorem we have

$$
\begin{array}{r}
\left|\iint_{\Omega} \rho \nabla \psi^{(1)} \cdot \nabla \psi_{j, n}^{(1)} d x d y+\int_{\Gamma_{0}} m \psi^{(4)} \psi_{j, n}^{(4)} d y\right| \leq \frac{C}{\left|\lambda_{j, n}\right|}\left\|\psi^{(1)}\right\|_{H^{3}(\Omega)} \\
\text { for } j^{2}+n^{2} \geq \lambda^{\star} .
\end{array}
$$


Similarly for $j^{2}+n^{2} \geq \lambda^{\star}$, one can easily prove

$$
\begin{aligned}
& \left|\iint_{\Omega} \psi^{(2)} \psi_{j, n}^{(2)} d x d y\right| \leq \frac{C}{\left|\lambda_{j, n}\right|}\left\|\psi^{(2)}\right\|_{H^{2}(\Omega)}, \\
& \left|\int_{\Gamma_{0}} \psi^{(3)} \psi_{j, n}^{(3)} d y\right| \leq \frac{C}{\left|\lambda_{j, n}\right|}\left\|\psi^{(3)}\right\|_{L^{2}(0,1)} .
\end{aligned}
$$

From (4.6), (4.7) and (4.9)-(4.10) we have

$$
\begin{array}{r}
\left|\eta_{j, n}^{(1)}\right| \leq C\left|\lambda_{j, n}\right|^{-2}\left[\left\|\psi^{(1)}\right\|_{H^{3}(\Omega)}+\left\|\psi^{(2)}\right\|_{H^{2}(\Omega)}+\left\|\psi^{(3)}\right\|_{L^{2}(0,1)}\right] \\
\text { for } j^{2}+n^{2} \geq \lambda^{\star} .
\end{array}
$$

Similarly one can get the same estimates of $(4.11)$ for $\eta_{j, n}^{(2)}$. Thus lemma 6 holds. Q.E.D.

Theorem 5. Assume that $k>0$. There is $\alpha^{\star}>0$ such that for every $d \leq \alpha^{\star}$, the following results hold

1) If the initial data $\vec{u}(0)=\left(\phi_{0}, \phi_{1}, \delta_{0}, \delta_{1}\right) \in \mathcal{H}$, then the energy of the system (1.1) decays to zero.

2) If the initial data $\vec{u}(0) \in \mathcal{D}(\mathcal{A})$ where $\phi_{0} \in H^{3}(\Omega)$ and $\phi_{1} \in H^{2}(\Omega)$ with $\frac{\partial \phi_{1}}{\partial \nu}=0 \quad$ a.e. on $\partial \Omega$, then the energy of the system (1.1) satisfies

$$
\mathcal{E}(t) \leq C t^{-1}\left[\left\|\phi_{0}\right\|_{H^{3}(\Omega)}^{2}+\left\|\phi_{1}\right\|_{H^{2}(\Omega)}^{2}+\left\|\delta_{0}\right\|_{L^{2}(0,1)}^{2}\right] \quad \text { for } t \geq 1
$$

Proof. We first claim

$$
\left\|e^{\left(\mathcal{A} \mathcal{P}_{\gamma^{\star}}\right) t}\right\| \leq C e^{-\hat{\lambda}^{\star} t}
$$

where $\mathcal{P}_{\gamma^{\star}}$ is the same as in (3.7) and $\hat{\lambda}^{\star}$ is the same as in (2.23). In fact, we have that $\left\|\left(\lambda-\mathcal{A} \mathcal{P}_{\gamma^{\star}}\right)^{-1}\right\| \leq C$ uniformly on $\lambda \geq 2\left\|\mathcal{A} \mathcal{P}_{\gamma^{\star}}\right\|$ since $\mathcal{A} \mathcal{P}_{\gamma^{\star}}$ is bounded by the proof of lemma 3 . We also have that $\left\|\left(\lambda-\mathcal{A} \mathcal{P}_{\gamma^{\star}}\right)^{-1}\right\| \leq C$ uniformly on $\left[-2\left\|\mathcal{A} \mathcal{P}_{\gamma^{\star}}\right\| i, 2\left\|\mathcal{A} \mathcal{P}_{\gamma^{\star}}\right\| i\right]$ which is compact and lies in the resolvent set of $\mathcal{A} \mathcal{P}_{\gamma^{\star}}$. Thus (4.13) follows from theorem 3 of Huang[4]. 
For 1$)$, let $\vec{u}(0)$ have the expansion in (3.7) by replacing $\vec{\psi}$ with $\vec{u}(0)$. Since $\mathcal{P}_{\gamma^{\star}} \mathcal{H}$ is an invariant subspace of $\mathcal{A}$, the solution of the evolution equation (1.6) is given by

$$
\vec{u}(t)=e^{\left(\mathcal{A} \mathcal{P}_{\gamma^{\star}}\right) t} \mathcal{P}_{\gamma^{\star}} \vec{\varphi}+\sum_{j^{2}+n^{2} \geq \lambda^{\star}}\left[\eta_{j, n}^{(1)} \frac{e^{\lambda_{j, n} t} \vec{\psi}_{j, n}}{\left\|\vec{\psi}_{j, n}\right\|_{\mathcal{H}}}+\eta_{j, n}^{(2)} \frac{e^{\bar{\lambda}_{j, n} t} \overline{\vec{\psi}}_{j, n}}{\left\|\vec{\psi}_{j, n}\right\|_{\mathcal{H}}}\right]
$$

For any $\epsilon>0$ given, by $((3.8)$ of $)$ theorem 4 we know that there is a constant $\omega$ such that

$$
\sum_{j^{2}+n^{2} \geq \omega}\left[\left|\eta_{j, n}^{(1)}\right|^{2}+\left|\eta_{j, n}^{(2)}\right|^{2}\right] \leq \frac{1}{2} \epsilon
$$

By lemma 2 and (4.13) there is $t^{\star}$ such that if $t \geq t^{\star}$, then

$$
\begin{aligned}
& \left\|e^{\left(\mathcal{A} \mathcal{P}_{\gamma^{\star}}\right) t} \mathcal{P}_{\gamma^{\star}} \vec{\varphi}\right\|_{\mathcal{H}}^{2}+\sum_{\lambda^{\star} \leq j^{2}+n^{2}<\omega}\left[\left|\eta_{j, n}^{(1)}\right|^{2}+\left|\eta_{j, n}^{(2)}\right|^{2}\right] e^{2 R e\left(\lambda_{j, n}\right) t} \\
& \leq C\left[e^{-\hat{\lambda}^{\star} t}+e^{-2 \kappa t}\right] \leq \frac{1}{2} \epsilon
\end{aligned}
$$

where $-\kappa=\sup \left\{\operatorname{Re}\left(\lambda_{j, n}\right) ; \quad \lambda^{\star} \leq j^{2}+n^{2}<\omega\right\}$. Thus by (3.8) of theorem 4 and (4.14)-(4.16) we have $\mathcal{E}(t)=\frac{1}{2}\|\vec{u}(t)\|_{\mathcal{H}}^{2} \leq C \epsilon$ for $t \geq t^{\star}$.

2) is a direct result of (4.13), lemma 6 and lemma 5 for $\beta=1$. Q.E.D.

Generally even if the initial data of the system (1.1) has more smoothness than in 2) of theorem 5 , one could not get higher decay rates than $t^{-1}$ for the energy of the system (1.1) due to the boundary integrals in (4.9) and (4.10). However we have the following corollary.

Corollary 1. Assume that $k>0, \phi_{0} \in H_{0}^{2 \beta+1}(\Omega), \phi_{1} \in H_{0}^{2 \beta}(\Omega)$ for any fixed positive integer $\beta$ and $\delta_{0}=\delta_{1}=0$. Then there is $\alpha^{\star}>0$ such that for every $d \leq \alpha^{\star}$ and any $\beta \geq 1$, the energy of the system (1.1) satisfies

$$
\mathcal{E}(t) \leq C t^{-(2 \beta-1)}\left[\left\|\phi_{0}\right\|_{H_{0}^{2 \beta+1}(\Omega)}^{2}+\left\|\phi_{1}\right\|_{H_{0}^{2 \beta}(\Omega)}^{2}\right] \quad \text { for } t \geq 1
$$


Proof. By the same argument as (4.9) and (4.10) we have

$$
\begin{array}{r}
\left|\eta_{j, n}^{(1)}\right|+\left|\eta_{j, n}^{(2)}\right| \leq C\left|\lambda_{j, n}\right|^{-2 \beta}\left[\left\|\phi_{0}\right\|_{H_{0}^{2 \beta+1}(\Omega)}+\left\|\phi_{1}\right\|_{H_{0}^{2 \beta}(\Omega)}\right] \\
\text { for } j^{2}+n^{2} \geq \lambda^{\star}
\end{array}
$$

in the expansion of $\vec{u}(0)$ as in (3.7). Thus corollary 1 holds by the same argument as in the proof of 2) of theorem $5 . \quad$ Q.E.D.

Finally with the techniques of interpolation spaces (cf. Bergh \& Löfströn[11] or Lions \& Magenes[12]) and the interpolation theorem (cf. Theorem 3.1 of "Chapter Premier" of Lions \& Peetre[13]), we shall prove that the energy of the system (1.1) decays at certain rational rates even though the initial data $\phi_{0}$ and $\phi_{1}$ in (1.1) is only slightly smoother than $\phi_{0} \in H^{1}(\Omega)$ and $\phi_{1} \in L^{2}(\Omega)$ respectively.

Theorem 6. Assume that $k>0, \phi_{0} \in H_{0}^{1+2 \theta}(\Omega)$ and $\phi_{1} \in H_{0}^{2 \theta}(\Omega)$ for certain $\theta \in(0,1), \delta_{0} \in L^{2}(0,1)$ and $\delta_{1}=0$. Then there is an $\alpha^{\star}>0$ such that for every $d<\alpha^{\star}$, the energy of the system (1.1) satisfies

$$
\mathcal{E}(t) \leq C(\theta) t^{-\theta}\left[\left\|\phi_{0}\right\|_{H_{0}^{1+2 \theta}(\Omega)}^{2}+\left\|\phi_{1}\right\|_{H_{0}^{2 \theta}(\Omega)}^{2}+\left\|\delta_{0}\right\|_{L^{2}(0,1)}^{2}\right], \quad t \geq 1
$$

Proof. Let $\mathcal{S}(t)$ denote the $C_{0^{-}}$contraction semigroup generated by the infinitesimal generator $\mathcal{A}$ (see proposition 1 ). By 2) of theorem 5 , we have

$$
\left\|\mathcal{S}(t)\left(0,0, \delta_{0}, 0\right)\right\|_{\mathcal{H}} \leq C t^{-\frac{1}{2}}\left\|\delta_{0}\right\|_{L^{2}(0,1)}, \quad t \geq 1
$$

We know that there is a sequence of $\left\{\phi_{0}^{(n)}\right\} \subset \mathcal{D}(\Omega)$ such that $\lim _{n \rightarrow \infty} \| \phi_{0}^{(n)}-$ $\phi_{0} \|_{H_{0}^{1+2 \theta}(\Omega)}=0$. Let $t \geq 1, n$ be fixed and $\Omega_{n} \subset \Omega$ be a smooth domain such that $\operatorname{supp}\left(\phi_{0}^{(n)}\right) \subset \Omega_{n}$. We define a linear operator $\tilde{T}: H_{0}^{1}\left(\Omega_{n}\right) \rightarrow \mathcal{H}$ by

$$
\tilde{T} u=\mathcal{S}(t)(\hat{u}, 0,0,0), \quad u \in H_{0}^{1}\left(\Omega_{n}\right),
$$


where $\hat{u}$ is an extension of $u$ from $\Omega_{n}$ to $\Omega$ by simply setting $\hat{u}=0$ in $\Omega_{n} \backslash \Omega$. Then by 2) of theorem 5 we have

$$
\begin{array}{rlrl}
\|\tilde{T} u\|_{\mathcal{H}} & \leq\|u\|_{H_{0}^{1}\left(\Omega_{n}\right)} & \forall u \in H_{0}^{1}\left(\Omega_{n}\right) \\
\|\tilde{T} u\|_{\mathcal{H}} \leq\|u\|_{H_{0}^{3}\left(\Omega_{n}\right)} & \forall u \in H_{0}^{3}\left(\Omega_{n}\right) .
\end{array}
$$

Let $\left(H_{0}^{s_{1}}\left(\Omega_{n}\right), H_{0}^{s_{2}}\left(\Omega_{n}\right)\right)_{\theta, 2}$ denote the interpolation space given by real $K$ method (there are several real interpolation methods used to define interpolation spaces, but in our case they are equivalent; cf. Theorem 3.12.1 of Bergh \& Löfströn[11] and Theorem 15.1 of Chapt. 1 of Lions \& Magenes[12]). Then we have $H_{0}^{1+2 \theta}\left(\Omega_{n}\right)=\left(H_{0}^{1}\left(\Omega_{n}\right), H_{0}^{3}\left(\Omega_{n}\right)\right)_{\theta, 2}$ (cf. Theorem 11.6 of Chapt. 1 of Lions $\&$ Magenes[12]). Since $(\mathcal{H}, \mathcal{H})_{\theta, 2}=\mathcal{H}$ (in sense of equivalent norms, cf. Theorem 3.4.1 of Bergh \& Löfströn[11]) for any $\theta$, it follows from the interpolation theorem and (4.21) that we have

$$
\left\|\tilde{T} \phi_{0}^{(n)}\right\|_{\mathcal{H}} \leq C(\theta) t^{-\frac{\theta}{2}}\left\|\phi_{0}^{(n)}\right\|_{H_{0}^{1+2 \theta}\left(\Omega_{n}\right)} .
$$

Let $n \rightarrow \infty$ in $(4.22)$ we have

$$
\left\|\mathcal{S}(t)\left(\phi_{0}, 0,0,0\right)\right\|_{\mathcal{H}} \leq C(\theta) t^{-\frac{\theta}{2}}\left\|\phi_{0}\right\|_{H_{0}^{1+2 \theta}(\Omega)} .
$$

Similarly we have

$$
\left\|\mathcal{S}(t)\left(0, \phi_{1}, 0,0\right)\right\|_{\mathcal{H}} \leq C(\theta) t^{-\frac{\theta}{2}}\left\|\phi_{1}\right\|_{H_{0}^{1+2 \theta}(\Omega)} .
$$

Thus (4.19) directly follows from the linearity of $\mathcal{S}(t),(4.20),(4.23)$ and $(4.24)$, and theorem 6 holds. Q.E.D.

Using the same argument of the proof of theorem 6 and corollary 2, we have

Corollary 2. Let $\beta \geq 1$ be an integer. Assume that $k>0, \phi_{0} \in H_{0}^{1+2 \beta \theta}(\Omega)$ and $\phi_{1} \in H_{0}^{2 \beta \theta}(\Omega)$ for certain $\theta \in(0,1)$, and $\delta_{0}=\delta_{1}=0$. Then there is $\alpha^{\star}>0$ such that for every $d \leq \alpha^{\star}$, the energy of the system (1.1) satisfies

$$
\mathcal{E}(t) \leq C(\theta, \beta) t^{-(2 \beta-1) \theta}\left[\left\|\phi_{0}\right\|_{H_{0}^{1+2 \beta \theta}(\Omega)}^{2}+\left\|\phi_{1}\right\|_{H_{0}^{2 \beta \theta}(\Omega)}^{2}\right], \quad t \geq 1 .
$$

Email address of second author: liu@math.umn.edu 


\section{References.}

[1] J.T. Beale, Spectral properties of an acoustic boundary condition, Indiana Univ. Math. J. 25. no. 9 (1976), 895-917.

[2] N. Dunford and J.T. Schwartz, Linear Operators. Part I \& II:, Interscience, New York (1963).

[3] J. K. Hale, Asymptotic Behavior of Dissipative Systems, American Math. Society, Providence, Rhode Island (1988).

[4] F.L. Huang, Characteristic conditions for exponential stability of linear dynamical systems in Hilbert spaces, Ann. of Diff. Equ. 1 (1983), 43-55.

[5] T. Kato, Perturbation Theory for Linear Operators, Springer-Verlag, New York (1980).

[6] W. Littman and L. Markus, Some recent results on control and stabilization of flexible structures, A.V.Balakrishnan and J.P.Zolesio ed, Optimization Software Inc. (1988), 151-161.

[7] W. Littman and L. Markus, Stabilization of a hybrid system of elasticity by feedback boundary damping, Ann. Math. Pura. Appl. 152 (1988), 281-330.

[8] S.D. Micu and E.Zuazua, Propriétés qualitatives d'un modèle hybride bidimensionnel intervenant dans le contrôle du bruit, C. R. Acad. Sci. Paris No 12 (1994), 1263-1268.

[9] S.D. Micu and E.Zuazua, Boundary controllability of a linear hybrid system arising in the control of noise, Preprint (1996).

[10]P.M. Morse and K.U. Ingard, Theoretical Acoustics, McGraw-Hill, New York (1968). 
[11]J. Bergh and J. Löfströn, Interpolation Spaces: an Introduction, SpringerVerlag, New York. (1976).

[12]J.L. Lions and E. Magenes, Problèms Aux Limites Non Homogènes Et Applications. vol I, Dunod, Paris (1968).

[13]J.L. Lions and J. Peetre, Sur une classe d'espaces d'interpolation, Inst. Hautes Études Sci. Publ. Math. 19 (1964), 5-68. 